\title{
Physical point simulation in 2+1 flavor lattice QCD
}

\author{
Yoshinobu Kuramashi* for PACS-CS Collaboration \\ Graduate School of Pure and Applied Sciences and Center for Computational Sciences, \\ University of Tsukuba, Tsukuba, Ibaraki 305-8571, Japan \\ E-mail: kuramasi@het.ph.tsukuba.ac.jp
}

We present preliminary results for a physical point simulation in 2+1 flavor lattice QCD with the nonperturbatively $O(a)$-improved Wilson quark action and the Iwasaki gauge action at $\beta=1.9$ on a $32^{3} \times 64$ lattice. The physical quark masses together with the lattice spacing are determined with $m_{\pi}, m_{K}$ and $m_{\Omega}$ as physical inputs. There are two key algorithmic ingredients to make possible the direct simulation at the physical point: One is the mass-preconditioned domain-decomposed HMC algorithm to reduce the computational cost. The other is the rewighting technique to adjust the hopping parameters exactly on the physical point. The physics results include the hadron spectrum, the pseudoscalar meson decay constants and the physical quark masses.

The XXVII International Symposium on Lattice Field Theory - LAT2009

July 26-31 2009

Peking University, Beijing, China

\footnotetext{
* Speaker.
} 


\section{Introduction}

The physical point simulation is a long-standing problem in lattice QCD because of the rapid growth of the computational cost with the up-down (ud) quark mass reduced toward its physical value. At present simulation points are typically restricted to $m_{\pi} \gtrsim 250 \mathrm{MeV}$. The most popular strategy to obtain estimates at the physical point is chiral extrapolation with the use of Chiral Perturbation Theory (ChPT) as a guiding principle. This strategy, however, has several problems: (i) It is numerically difficult to precisely trace the logarithmic quark mass dependence of the physical quantities predicted by ChPT. (ii) It may not be always possible to resort to ChPT as a good guiding principle for chiral extrapolation. (iii) The kinematics is changed as the quark mass increases. A typical example is the $\rho \rightarrow \pi \pi$ decay which is not allowed for the increased ud quark mass away from the physical value. (iv) Our final destination is to incorporate the different up and down quark masses, whose isospin breaking effects are so tiny that the reliable evaluation would be difficult by the chiral extrapolation method.

In this report we present the preliminary results of the physical point simulation which has been pursued as the PACS-CS project based on the PACS-CS (Parallel Array Computer System for Computational Sciences) computer with a peak speed of 14.3 Tflops developed at University of Tsukuba!1 2, 3]. The simulation is carried out with the nonperturbatively $O(a)$-improved Wilson quark action [4 and the Iwasaki gauge action [5] on a $(3 \mathrm{fm})^{3}$ box at the lattice spacing of $a=$ $0.09009(43) \mathrm{fm}$. There are two types of problems in the physical point simulation. Firstly, we need to reduce the computational cost which rapidly increases as the ud quark mass decreases. This difficulty is overcome thanks to the domain-decomposed HMC (DDHMC) algorithm [6] with the mass-preconditioning [7, 8]. In Refs. [9 10] this algorithm was successfully applied to investigate the chiral behaviors of the hadron masses including both the mesons and the baryons, where the pion mass covers from $156 \mathrm{MeV}$ to $702 \mathrm{MeV}$. The second problem is the fine-tuning of the quark masses on the physical point after we reach around the physical point. This task is accomplished with the reweighting technique. We explain the details of the method and present the physics results on the physical point without any interpolation or extrapolation.

\section{Simulation parameters and algorithm}

We employ the same parameters as in the previous work 9 : $32^{3} \times 64$ lattice at $\beta=1.90$ with $c_{\mathrm{SW}}=1.715$ [4]. We choose $\left(\kappa_{\mathrm{ud}}, \kappa_{\mathrm{s}}\right)=(0.13778500,0.1366000)$ which was supposed to be the physical point based on the analysis of the previous results [9]. The physical ud and s quark masses and the lattice spacing are determined by $m_{\pi}, m_{K}$ and $m_{\Omega}$. We calculate hadronic observables at every 100 trajectories.

The base algorithm for the ud quark is the DDHMC algorithm [6 which makes a geometric separation of the ud quark determinant into the UV and the IR parts with the domain-decomposition of the full lattice into small blocks. Our choice for the block size is $8^{4}$. In this work we implement twofold-mass-preconditioned DDHMC (MP2 DDHMC) algorithm which is an extension of the mass-preconditioned DDHMC (MPDDHMC) algorithm employed in Ref. [9] to simulate the lightest ud quark mass at $\left(\kappa_{\mathrm{ud}}, \kappa_{\mathrm{s}}\right)=(0.13781000,0.1364000)$ [9]. In MP ${ }^{2}$ DDHMC algorithm the IR force $F_{\mathrm{IR}}$ is split into $\tilde{F}_{\mathrm{IR}}, F_{\mathrm{IR}}^{\prime}$ and $F_{\mathrm{IR}}^{\prime \prime}$. This decomposition is controlled by two additional 
hopping parameters $\kappa_{\mathrm{ud}}^{\prime}=\rho_{1} \kappa$ and $\kappa_{\mathrm{ud}}^{\prime \prime}=\rho_{1} \rho_{2} \kappa . \quad \tilde{F}_{\mathrm{IR}}$ is derived from the action preconditioned with $\kappa_{\mathrm{ud}}^{\prime}$. The ratio of two preconditioners with $\kappa_{\mathrm{ud}}^{\prime}$ and $\kappa_{\mathrm{ud}}^{\prime \prime}$ gives $F_{\mathrm{IR}}^{\prime} . F_{\mathrm{IR}}^{\prime \prime}$ is for the heaviest preconditioners with $\kappa_{\mathrm{ud}}^{\prime \prime}$. We find the following relative magnitudes for the force terms:

$$
\left\|F_{\mathrm{g}}\right\|:\left\|F_{\mathrm{UV}}\right\|:\left\|F_{\mathrm{IR}}^{\prime \prime}\right\|:\left\|F_{\mathrm{IR}}^{\prime}\right\|:\left\|\tilde{F}_{\mathrm{IR}}\right\| \approx 16: 4: 1: 1 / 7: 1 / 60 .
$$

with $\rho_{1}=0.9995$ and $\rho_{2}=0.9900$. We choose $\left(N_{0}, N_{1}, N_{2}, N_{3}, N_{4}\right)=(4,4,2,4,4)$ for the associated step sizes in the multiple time scale integration scheme[11]: $\delta \tau_{\mathrm{g}}=\tau /\left(N_{0} N_{1} N_{2} N_{3} N_{4}\right), \delta \tau_{\mathrm{UV}}=$ $\tau /\left(N_{1} N_{2} N_{3} N_{4}\right), \delta \tau_{\text {IR }}^{\prime \prime}=\tau /\left(N_{2} N_{3} N_{4}\right), \delta \tau_{\text {IR }}^{\prime}=\tau /\left(N_{3} N_{4}\right), \delta \tilde{\tau}_{\text {IR }}=\tau / N_{4}$ with $\tau=0.25$. The replay trick 612] is not incorporated. For the inversion of the Wilson-Dirac operator during the molecular dynamics (MD) steps we implement the same algorithmic techniques as in the run at $\left(\kappa_{\mathrm{ud}}, \kappa_{\mathrm{s}}\right)=$ $(0.13781000,0.1364000)$ in the previous work 9 .

The strange quark is simulated with the UV-filtered PHMC (UVPHMC) algorithm,13, 14, 15, [16] where the action is UV-filtered [17] after the even-odd site preconditioning without domaindecomposition. The polynomial order is $N_{\text {poly }}=220$ in our choice. We set the step size as $\delta \tau_{\mathrm{s}}=$ $\delta \tau_{\mathrm{IR}}^{\prime \prime}$ according to our observation $\left\|F_{\mathrm{s}}\right\| \approx\left\|F_{\mathrm{IR}}^{\prime \prime}\right\|$. This algorithm is made exact by correcting the polynomial approximation with the global Metropolis test [18 at the end of each trajectory.

\section{REWEIGHTING METHOD}

\subsection{Formalism}

Let us consider evaluating $\left\langle\mathscr{O}[U]\left(\kappa_{\mathrm{ud}}^{\prime}, \kappa_{\mathrm{s}}^{\prime}\right)\right\rangle_{\left(\kappa_{\mathrm{ud}}^{\prime}, \mathrm{\kappa}_{\mathrm{s}}^{\prime}\right)}$, which is the expectation value of a physical observable $\mathscr{O}$ at the target hopping parameters $\left(\kappa_{\mathrm{ud}}^{\prime}, \kappa_{\mathrm{s}}^{\prime}\right)$, using configuration samples generated at the original hopping parameters $\left(\kappa_{\mathrm{ud}}, \kappa_{\mathrm{s}}\right)$. We assume that $\rho_{\mathrm{ud}} \equiv \kappa_{\mathrm{ud}} / \kappa_{\mathrm{ud}}^{\prime} \simeq 1$ and $\rho_{\mathrm{s}} \equiv \kappa_{\mathrm{s}} / \kappa_{\mathrm{s}}^{\prime} \simeq 1$. The expectation value is rewritten as follows:

$$
\begin{aligned}
& \left\langle\mathscr{O}[U]\left(\kappa_{\mathrm{ud}}^{\prime}, \kappa_{\mathrm{s}}^{\prime}\right)\right\rangle_{\left(\kappa_{\mathrm{ud}}^{\prime}, \kappa_{\mathrm{s}}^{\prime}\right)} \\
= & \frac{\int \mathscr{D} U \mathscr{O}[U]\left(\kappa_{\mathrm{ud}}^{\prime}, \kappa_{\mathrm{s}}^{\prime}\right)\left|\operatorname{det}\left[D_{\kappa_{\mathrm{ud}}^{\prime}}[U]\right]\right|^{2} \operatorname{det}\left[D_{\kappa_{\mathrm{s}}^{\prime}}[U]\right] \mathrm{e}^{-S_{g}[U]}}{\int \mathscr{D} U\left|\operatorname{det}\left[D_{\kappa_{\mathrm{ud}}^{\prime}}[U]\right]\right|^{2} \operatorname{det}\left[D_{\mathrm{\kappa}_{\mathrm{s}}^{\prime}}[U]\right] \mathrm{e}^{-S_{g}[U]}} \\
= & \frac{\int \mathscr{D} U \mathscr{O}[U]\left(\kappa_{\mathrm{ud}}^{\prime}, \kappa_{\mathrm{s}}^{\prime}\right)\left|\operatorname{det}\left[\frac{D_{\kappa_{\mathrm{s}}^{\prime}}[U]}{D_{\kappa_{\mathrm{ud}}}[U]}\right]\right|^{2} \operatorname{det}\left[\frac{D_{\kappa_{\mathrm{s}}^{\prime}}[U]}{D_{\mathrm{\kappa}_{\mathrm{s}}[U]}[U]}\right]\left|\operatorname{det}\left[D_{\kappa_{\mathrm{ud}}}[U]\right]\right|^{2} \operatorname{det}\left[D_{\kappa_{\mathrm{s}}}[U]\right] \mathrm{e}^{-S_{g}[U]}}{\int \mathscr{D} U\left|\operatorname{det}\left[D_{\kappa_{\mathrm{ud}}^{\prime}}[U]\right]\right|^{2} \operatorname{det}\left[D_{\kappa_{\mathrm{s}}^{\prime}}[U]\right] \mathrm{e}^{-S_{g}[U]}} \\
= & \frac{\left\langle\mathscr{O}[U]\left(\kappa_{\mathrm{ud}}^{\prime}, \kappa_{\mathrm{s}}^{\prime}\right) R_{\mathrm{ud}}[U] R_{\mathrm{s}}[U]\right\rangle_{\left(\kappa_{\mathrm{ud}}, \kappa_{\mathrm{s}}\right)}}{\left\langle R_{\mathrm{ud}}[U] R_{\mathrm{s}}[U]\right\rangle_{\left(\kappa_{\mathrm{ud}}, \kappa_{\mathrm{s}}\right)}}
\end{aligned}
$$

The reweighting factors are defined as

$$
R_{\mathrm{ud}}[U]=\left|\operatorname{det}\left[W[U]\left(\rho_{\mathrm{ud}}\right)\right]\right|^{2}, \quad R_{\mathrm{s}}[U]=\operatorname{det}\left[W[U]\left(\rho_{\mathrm{s}}\right)\right]
$$

with

$$
W[U]\left(\rho_{q}\right) \equiv \frac{D_{\kappa_{q}^{\prime}}[U]}{D_{\kappa_{q}}[U]}, \quad D_{\kappa_{q}}[U]=1+\kappa_{q}(T+M) \quad(q=\mathrm{ud}, \mathrm{s})
$$

where $T$ the local clover term including the nonperturbative $c_{\mathrm{SW}}$ and $M$ the hopping matrix. The above expression (3.1) demands us to evaluate the reweighting factors $R_{\mathrm{ud}}[U]$ and $R_{\mathrm{S}}[U]$ on each configuration. 


\subsection{Evaluation of reweighting factors}

The reweighting factor $R_{\mathrm{ud}}[U]$ can be evaluated with a stochastic method. Introducing a complex bosonic field $\eta$, whose spin and color indices are suppressed here, the determinant of $W$ is expressed as

$$
\begin{aligned}
R_{\mathrm{ud}}[U] & =\frac{\int \mathscr{D} \eta^{\dagger} \mathscr{D} \eta \mathrm{e}^{-\left|W^{-1}[U]\left(\rho_{\mathrm{ud}}\right) \eta\right|^{2}}}{\int \mathscr{D} \eta^{\dagger} \mathscr{D} \eta \mathrm{e}^{-|\eta|^{2}}} \\
& =\frac{\int \mathscr{D} \eta^{\dagger} \mathscr{D} \eta \mathrm{e}^{-\left|W^{-1}[U]\left(\rho_{\mathrm{ud}}\right) \eta\right|^{2}+|\eta|^{2}-|\eta|^{2}}}{\int \mathscr{D} \eta^{\dagger} \mathscr{D} \eta \mathrm{e}^{-|\eta|^{2}}} \\
& =\left\langle\mathrm{e}^{-\left|W^{-1}[U]\left(\rho_{\mathrm{ud}}\right) \eta\right|^{2}+|\eta|^{2}}\right\rangle_{\eta},
\end{aligned}
$$

where $\langle\cdots\rangle_{\eta}$ means the expectation value with respect to $\eta$. Given a set of $\eta^{(i)}\left(i=1, \ldots, N_{\eta}\right)$ which are random noises generated according to the Gaussian distribution, the reweighting factor is evaluated as

$$
R_{\mathrm{ud}}[U]=\lim _{N_{\eta} \rightarrow \infty} \frac{1}{N_{\eta}} \sum_{i=1}^{N_{\eta}} \mathrm{e}^{-\left|W^{-1}[U]\left(\rho_{\mathrm{ud}}\right) \eta\right|^{2}+|\eta|^{2}} .
$$

The ratio $W^{-1}$ is further simplified as follows:

$$
W^{-1}[U]\left(\rho_{\mathrm{ud}}\right)=\rho_{\mathrm{ud}}+\left(1-\rho_{\mathrm{ud}}\right) D_{\kappa_{\mathrm{ud}}^{\prime}}^{-1}[U]
$$

with $D_{\kappa_{\text {ud }}}[U]=\rho_{\text {ud }} D_{\kappa_{\text {ud }}^{\prime}}[U]+\left(1-\rho_{\text {ud }}\right)$. We just need $D_{\kappa_{\text {ud }}^{\prime}}^{-1}$ to calculate $W^{-1}$.

For the strange quark we assume that $\operatorname{det} W[U]\left(\rho_{\mathrm{s}}\right)$ is positive. The corresponding reweighting factor is evaluated as

$$
R_{\mathrm{s}}[U]=\frac{\int \mathscr{D} \eta^{\dagger} \mathscr{D} \eta \mathrm{e}^{-\left|W^{-1 / 2}[U]\left(\rho_{\mathrm{s}}\right) \eta\right|^{2}}}{\int \mathscr{D} \eta^{\dagger} \mathscr{D} \eta \mathrm{e}^{-|\eta|^{2}}}=\left\langle\mathrm{e}^{-\left|W^{-1 / 2}[U]\left(\rho_{\mathrm{s}}\right) \eta\right|^{2}+|\eta|^{2}}\right\rangle_{\eta}
$$

With the assumption of $\rho_{\mathrm{s}} \simeq 1$ we expect that $W[U]\left(\rho_{\mathrm{s}}\right)$ is so close to the identity matrix that the eigenvalues are enclosed by a unit circle centered at $(1,0)$ in the complex plane. In this case we can evaluate $W^{-1 / 2}[U]\left(\rho_{\mathrm{s}}\right) \eta$ by the Taylor expansion around identity.

We first parametrize $W^{-1}[U]\left(\rho_{\mathrm{s}}\right)$ as

$$
W^{-1}[U]\left(\rho_{\mathrm{s}}\right)=\rho_{\mathrm{s}}+\left(1-\rho_{\mathrm{s}}\right)\left(D_{\mathcal{K}_{\mathrm{s}}^{\prime}}[U]\right)^{-1}=1-\left(1-\rho_{\mathrm{s}}\right)\left(1-\left(D_{\kappa_{\mathrm{s}}^{\prime}}[U]\right)^{-1}\right)=1-X[U]\left(\rho_{\mathrm{s}}\right),
$$

where $\left|1-\rho_{\mathrm{s}}\right| \ll 1$ and $\left\|X[U]\left(\rho_{\mathrm{s}}\right)\right\|<1$. We employ a recursive expression for the Taylor expansion of $W^{-1 / 2}[U]\left(\rho_{\mathrm{s}}\right) \eta[15]$ :

$$
\begin{aligned}
W^{-1 / 2} \eta & =\sum_{j=0}^{N} c_{j} X^{j} \eta \\
& =c_{0}\left[\eta+\frac{c_{1}}{c_{0}} X\left[\eta+\frac{c_{2}}{c_{1}} X\left[\eta+\frac{c_{3}}{c_{2}} X\left[\cdots\left[\eta+\frac{c_{N-1}}{c_{N-2}} X\left[\eta+\frac{c_{N}}{c_{N-1}} X \eta\right]\right]\right]\right]\right]\right],
\end{aligned}
$$

where the arguments $[U]\left(\rho_{\mathrm{s}}\right)$ for the matrices are suppressed. The coefficients are given by $c_{j} / c_{j-1}=$ $1-3 /(2 j)$ with $c_{0}=1$. The advantage of the recursive procedure is to reduce the round-off errors in the summation from the lower-order to the higher-order contributions in the Taylor expansion. 
The fluctuations in the stochastic evaluation of $R_{\mathrm{ud}}[U]$ and $R_{\mathrm{S}}[U]$ can be reduced by the determinant breakup technique [19]. After dividing the interval between $\kappa_{q}$ and $\kappa_{q}^{\prime}$ into the $N_{B}$ subintervals with $\Delta_{q}=\left(\kappa_{q}^{\prime}-\kappa_{q}\right) / N_{B}$, we obtain

$$
\operatorname{det}\left[W^{-1}[U]\left(\rho_{\mathrm{q}}\right)\right]=\operatorname{det}\left[W^{-1}[U]\left(\frac{\kappa_{q}+\Delta_{q}}{\kappa_{q}}\right)\right] \cdots \operatorname{det}\left[W^{-1}[U]\left(\frac{\kappa_{q}^{\prime}}{\kappa_{q}+\left(N_{B}-1\right) \Delta_{q}}\right)\right]
$$

where each determinant in the right hand side is evaluated with the different sets of $\eta$.

\subsection{Parameters and results for reweighting factors}

Our choice of the target hopping parameter is $\left(\kappa_{\mathrm{ud}}^{\prime}, \kappa_{\mathrm{s}}^{\prime}\right)=(0.13779625,0.1366225)$. The subintervals for the determinant breakup are $\Delta_{\mathrm{ud}}=(0.13779625-0.13778500) / 3$ for the ud quark and $\Delta_{\mathrm{s}}=(0.1366225-0.1366000)$ for the s quark. Each piece of the divided determinant is evaluated stochastically employing 10 sets of $\eta$.

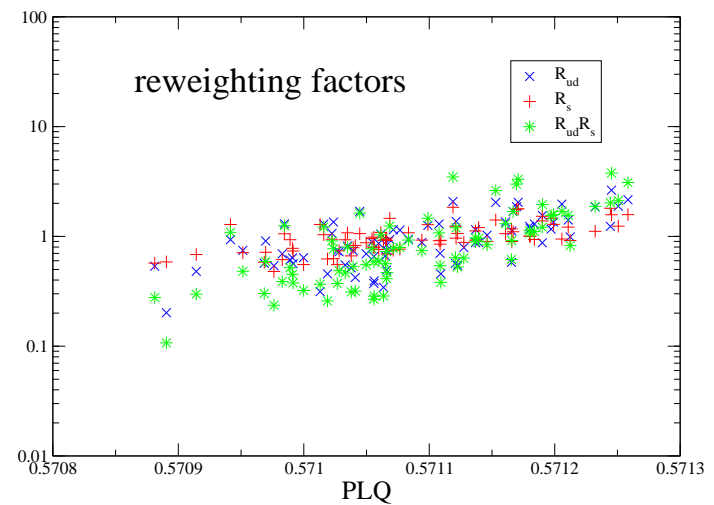

Figure 1: Reweighting factors as a function of plaquette value.

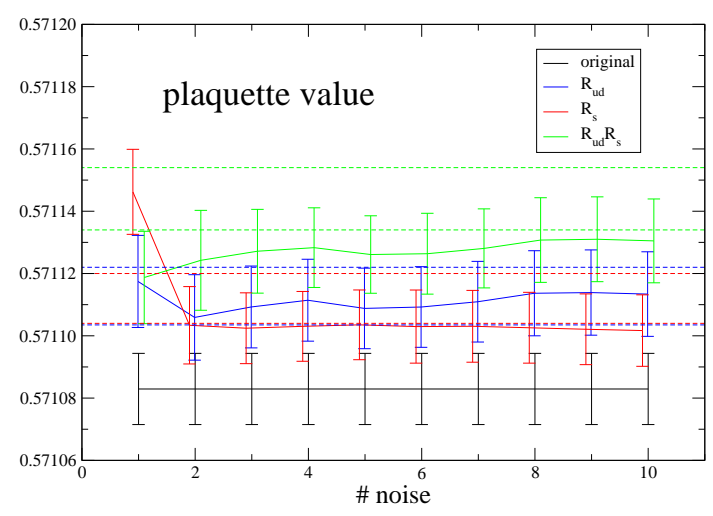

Figure 2: Reweighted plaquette values with $R_{\mathrm{ud}}$ and $R_{\mathrm{S}}$.

In Fig. 1 we plot the reweighting factors as a function of the plaquette value on each configuration, where we normalize the reweighting factors as $\left\langle R_{\mathrm{ud}, \mathrm{s}}\right\rangle=1$ and $\left\langle R_{\mathrm{ud}} R_{\mathrm{s}}\right\rangle=1$. The fluctuations of $R_{\mathrm{ud}}$ and $R_{\mathrm{S}}$ are within a factor of ten. Their product shows slightly amplified fluctuations. An important observation is a clear correlation between the reweighting factors and the plaquette value: The former increases as the latter becomes larger. Thanks to this correlation the distribution of the plaquette value at $\left(\kappa_{\mathrm{ud}}^{\prime}, \kappa_{\mathrm{s}}^{\prime}\right)=(0.13779625,0.1366225)$ is moved in the positive direction. This is the expected behavior, because the target hopping parameters are larger than the original ones. The situation is quantitatively illustrated in Fig. 2, where the reweighted plaquette values are plotted as a function of the number of noise. The results look converged once the number of noise goes beyond four. The horizontal lines indicate the expected plaquette values obtained by extrapolating the data at $\kappa_{\mathrm{ud}} \geq 0.13754000$ in Ref. [9] and $\left(\kappa_{\mathrm{ud}}, \kappa_{\mathrm{s}}\right)=(0.13778500,0.1366000)$ in this work. The reweighted results are consistent with the expectation values within error bars.

\section{Physics results}

In Fig. 3 we plot the kaon effective masses with the reweighting factors. The partially quenched result (PQ) shows lighter effective masses than the original case, which are further reduced by the 
reweighting effects $(\mathrm{PQ}+\mathrm{RW})$. Similar observation is obtained in other hadron channels. Figure 4 compares the measured hadron masses normalized by $m_{\Omega}$ with the experimental values. We employ $m_{\pi}, m_{K}, m_{\Omega}$ as physical inputs to determine $m_{\mathrm{ud}}, m_{\mathrm{s}}, a^{-1}$. The values of $m_{\pi}, m_{K}, m_{\Omega}$, which are sizably away from the experimental ones at the original simulation point (black symbols), are properly tuned on the physical point within error bars after the reweighting procedure. For other hadron masses the deviation from the experimental value is at most $5 \%$. An increasingly larger deviation observed for lighter baryons may be due to finite-size effects. The $\rho$ meson data is omitted in Fig. 4 since we do not observe a plateau for the $\rho$ meson effective mass. This may be due to the resonance effects, which we leave for future analyses.

The quark masses and the lattice cutoff are determined as $m_{\mathrm{ud}}^{\overline{\mathrm{MS}}}(2 \mathrm{GeV})=3.10(27)$ and $m_{\mathrm{s}}^{\overline{\mathrm{MS}}}(2$ $\mathrm{GeV})=96.31(37)$ with $a^{-1}=2.190(10) \mathrm{GeV}$, where we employ a nonperturbative renormalization factor obtained by the Schrödinger functional method[20]. The quark mass results are comparable to the recent estimate in the literature. For the pseudoscalar meson decay constants we obtain $f_{\pi}=124.0(7.2) \mathrm{MeV}$ and $f_{K}=165.5(3.2) \mathrm{MeV}$. employing the nonperturbative renormalization factor [20]. These values should be compared with the experimental ones: $f_{\pi}=130.4 \pm 0.04 \pm 0.2$ $\mathrm{MeV}$ and $f_{K}=155.5 \pm 0.2 \pm 0.8 \pm 0.2 \mathrm{MeV}[21]$. The deficit found in $f_{\pi}$ may be caused by the finite size effects, whose magnitude is expected to be about $4 \%$ on a $(3 \mathrm{fm})^{3}$ box at the physical point based on the NLO ChPT analyses [22, 9 .

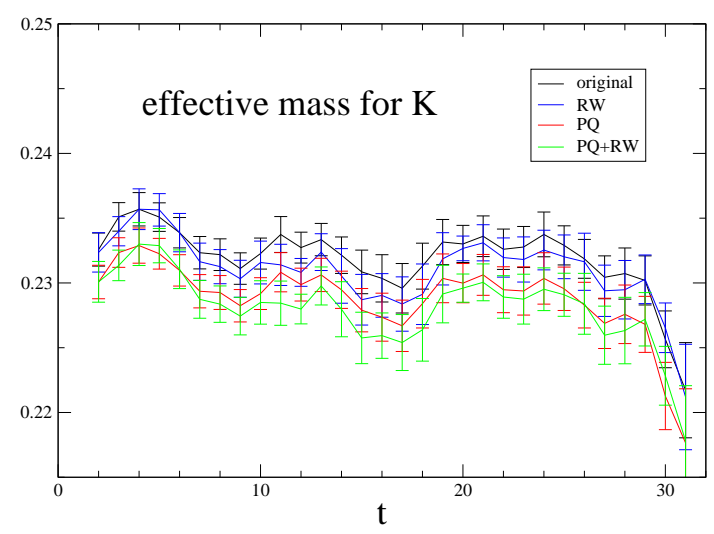

Figure 3: Kaon effective masses with reweighting (RW) and partially quenching (PQ) effects.

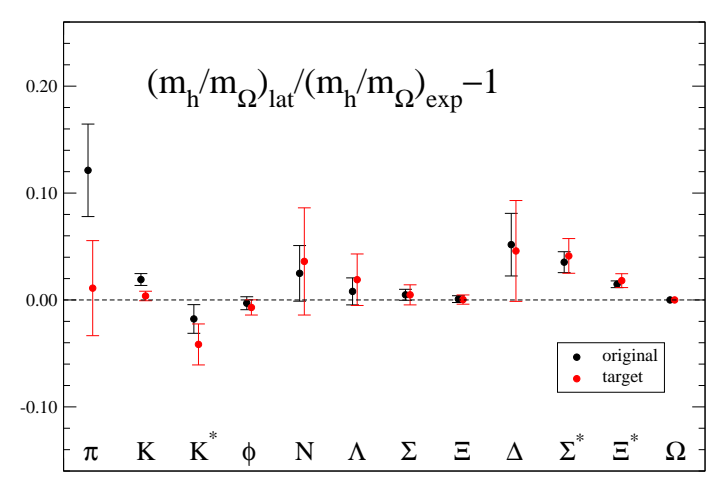

Figure 4: Hadron masses in comparison with experimental values.

\section{Acknowledgments}

Numerical calculations for the present work have been carried out on the PACS-CS computer under the "Interdisciplinary Computational Science Program" of Center for Computational Sciences, University of Tsukuba. A part of the code development has been carried out on Hitachi SR11000 at Information Media Center of Hiroshima University. This work is supported in part by Grants-in-Aid for Scientific Research from the Ministry of Education, Culture, Sports, Science and Technology (Nos. 16740147, 17340066, 18104005, 18540250, 18740130, 19740134, 20105002, 20340047, 20540248, 20740123, 20740139 ). 


\section{References}

[1] PACS-CS Collaboration, S. Aoki et al., PoS LAT2005, 111 (2005).

[2] PACS-CS Collaboration, A. Ukawa et al., PoS LAT2006, 039 (2006).

[3] T. Boku et al., Proceedings of CCGRID 2006, pp.233 (2006).

[4] CP-PACS and JLQCD Collaborations, S. Aoki et al., Phys. Rev. D73, 034501 (2006).

[5] Y. Iwasaki, preprint, UTHEP-118 (Dec. 1983), unpublished.

[6] M. Lüscher, JHEP 0305, 052 (2003); Comput. Phys. Commun. 165, 199 (2005).

[7] M. Hasenbusch, Phys. Lett. B519, 177 (2001).

[8] M. Hasenbusch and K. Jansen, Nucl. Phys. B659, 299 (2003).

[9] PACS-CS Collaboration, S. Aoki et al., Phys. Rev. D79, 034503 (2009).

[10] PACS-CS Collaboration, K.-I. Ishikawa et al., Phys. Rev. D80, 054502 (2009).

[11] J. C. Sexton and D. H. Weingarten, Nucl. Phys. B380, 665 (1992).

[12] A. Kennedy, Nucl. Phys. B (Proc. Suppl.) 140, 190 (2005).

[13] Ph. de Forcrand and T. Takaishi, Nucl. Phys. Proc. Suppl. 53, 968 (1997).

[14] R. Frezzotti and K. Jansen, Phys. Lett. B402, 328 (1997); Nucl. Phys. B555, 395 (1999); Nucl. Phys. B555, 432 (1999).

[15] JLQCD Collaboration, S. Aoki et al., Phys. Rev. D65, 094507 (2002).

[16] PACS-CS Collaboration, K.-I. Ishikawa et al., PoS LAT2006, 027 (2006).

[17] C. Alexandrou, P. de Forcrand, M. D’Elia and H. Panagopoulos, Phys. Rev. D61, 074503 (2000); Nucl. Phys. Proc. Suppl. 83, 765 (2000) P. de Forcrand, Nucl. Phys. Proc. Suppl. 73, 822 (1999).

[18] A. Boriçi and P. de Forcrand, Nucl. Phys. B454, 645 (1995); A. Borrelli, P. de Forcrand and A. Galli, Nucl. Phys. B477, 809 (1996); P. de Forcrand and A. Galli, arXiv:hep-lat/9603011; A. Galli and P. de Forcrand, Nucl. Phys. Proc. Suppl. 53, 956 (1997).

[19] A. Hasenfratz, R. Hoffmann and S. Schaefer, Phys. Rev. D78, 014515 (2008).

[20] PACS-CS Collaboration, Y. Taniguchi et al., PoS LATTICE2009, 208 (2009).

[21] C. Amsler et al. (Particle Data Group), Phys. Lett. B667, 1 (2008).

[22] G. Colangelo, S. Dürr and C. Haefeli, Nucl. Phys. B721, 136 (2005). 